\title{
Multi-Omics Characterization and Origin Assessment of Bilateral Lung Adenoid Cystic Carcinoma: A Case Report
}

This article was published in the following Dove Press journal: Cancer Management and Research

\author{
Minhua $\mathrm{Ye}^{1, *^{*}}$ \\ Sijia $\operatorname{Ren}^{2, *^{*}}$ \\ Chunguo Wang' \\ Xiaoshun Shi iD ${ }^{3}$ \\ Jianfei Shen' \\ 'Department of Cardiothoracic Surgery, \\ Taizhou Hospital of Zhejiang Province \\ Affiliated to Wenzhou Medical University, \\ Wenzhou, 317000, People's Republic of \\ China; ${ }^{2}$ Department of Cardiothoracic \\ Surgery, Taizhou Hospital of Zhejiang \\ Province Affiliated to Zhejiang University \\ School of Medicine, Hangzhou, 310000 , \\ People's Republic of China; ${ }^{3}$ Department \\ of Thoracic Surgery, Nafang Hospital, \\ Southern Medical University, Guangzhou, \\ 510515, People's Republic of China \\ *These authors contributed equally to \\ this work
}

Correspondence: Jianfei Shen Department of Cardiothoracic Surgery Taizhou Hospital of Zhejiang Province Affiliated to Wenzhou Medical University, Wenzhou, 317000, People's Republic of China

Email jianfei05I@I63.com

Xiaoshun Shi

Department of Thoracic Surgery, Nanfang Hospital, Southern Medical University, No. 1838 North Guangzhou Avenue, Guangzhou, 510515, People's Republic of China

Email sxs917@I63.com
Background: Primary adenoid cystic carcinoma (ACC) of the lung, which arises from the bronchial gland and is rare, accounting for only $0.04-0.2 \%$ of all primary lung tumors. The genetic profiling of bilateral ACC of unknown primary site and application in postoperative decision-making are less reported.

Case Presentation: A 57-year-old male with a smoking history of over 30 years and multiple nodules in both lungs was present to our department. After assessing the bilateral solid nodules in his Positron Emission Tomography-Computed Tomography (PET/CT) scan, malignant lesions at the left lower lung, right lower lung, and right middle lung are suspected. Sequential selective video-assisted thoracoscopic surgeries (VATS) were performed. A genetic alteration test of 425 cancer-related genes and global gene expression profile of the specimens revealed intrapulmonary metastasis existed. The patient was followed up for three years without recurrence and tissue mutations in liquid biopsy.

Conclusion: We present a way of omics-based multiple pulmonary lesions origin assessment, facilitating post-operative differential diagnosis and treatment decision for difficult cases.

Keywords: adenoid cystic carcinoma, next-generation sequencing, lung cancers of unknown primary site, PIK3R1

\section{Background}

Adenoid cystic carcinoma (ACC) is a malignant tumor that often occurs in the salivary glands, but it can also originate in breast, skin, upper digestive tract and lungs. ${ }^{1,2}$ According to the World Health Organization (WHO) classification of lung cancer, salivary gland tumors can be divided into mucoepidermoid carcinoma, adenoid cystic carcinoma, epithelial-myoepithelial carcinoma and pleomorphic carcinoma. ${ }^{3}$ Primary adenoid cystic carcinoma of the lung, which arises from the bronchial gland, is rare and accounts for only $0.04-0.2 \%$ of all primary lung tumors. ${ }^{4,5}$ Because of its low incidence, its clinicopathological features, mutational and gene expression profile, treatment and long-term survival have not been fully clarified. Studies have shown that more than $90 \%$ of ACC occurs in the trachea and main bronchus, and there are even fewer cases of peripheral lung ACC, in subsegmental bronchi. ${ }^{6}$ Routine pathological examination of $\mathrm{ACC}$, those in peripheral lungs with multiple lesions in both lungs sometimes can not be distinguished between multiple primary and metastatic lungs. For multiple synchronous ACCs in lungs, differentiation between multiple primary lesions and intrapulmonary metastasis is often a conundrum for post-operative staging and management. 


\section{Case Report}

A 57-year-old male with a smoking history of over 30 years and multiple nodules in both lungs was present to our department. After assessing the bilateral solid nodules in his Positron Emission Tomography-Computed Tomography (PET/CT) scan, malignant lesions at the left lower lung, right lower lung, and right middle lung are suspected. Without surgical contraindication, wedge resection by video-assisted thoracoscopic surgery (VATS) was first applied to the left lower lobe's nodule. The postoperative pathological of the left lower nodule (LLL) confirmed adenoid cystic carcinoma (ACC). Two months later, the procedure was used again to nodules in the right middle lobe (RML) and right lower lobe (RLL). Adenoid cystic carcinoma was confirmed in all lesions. Unfortunately, the pathological distinction between primary and metastatic lesions is unclear, impeding personalised decision of postoperative treatment and follow-up plans.

At first, clinical approved 425 cancer-relevant genes capture-based NGS test was done for all three pulmonary lesions (GeneseeqPrime) and genomic alterations among the lesions were compared (Table 1, Table S1). The oncoplot for genetic alterations show that $M C L 1$ gene amplification was detected in the LLL lesion. For the shared frameshift mutation in exon 2 of PIK3Rl gene p. I68Yfs*8 were detected in LLL and RML lesions, it implies a role in tumorigenesis and metastasis from left to right side (Figure 1).

Recently, it has been reported that gene expression profiling can distinguish primary and metastatic lesions in varied cancer types. ${ }^{7-9}$ To further assess the origin and the relationship of these lesions, we profiled their global gene expression using the Affymetrix GeneChip ${ }^{\circledR}$ PrimeViewTM Human Gene Expression Array. Transcriptomic data from resected paracancerous lung, LLL, RML, and RLL tissue revealed their intrinsic relationship. A total of 2262 differential genes against control tissue are shared among three ACC samples, but the number of differential genes is imbalanced between the left and right lung (Figure 2A). Further coexpression analysis of gene expression within groups showed distinct expression patterns between normal and cancer tissue while a close relationship among cancer samples is observed, indicating intrinsic similarities among ACC samples (Figure 2B). Next, principal component analysis on differentially expressed genes further confirmed that the transcriptomic similarity is transitionally higher between RML and RLL compared with RML and LLL, suggesting right-side lesion could be derived from the left-side lesion (Figure 2C). The Kyoto Encyclopedia of Genes and Genomes (KEGG) enrichment analysis on the top 200 differential genes by clusterProfiler revealed metastasis-associated cell pathways (focal adhesion, PI3K-Akt signaling pathway, and ECM-receptor interaction) are progressively activated from left tumor tissue to right tumor tissues (Figure 2D). We also observed similar transition in Gene Ontology overlapping based on the top 10 terms of biological process, molecular function and cellular component (Table 2). Taken together, the mutation characteristic, gene expression similarity, and cell signaling shift indicate that this patient had a left-side primary peripheral pulmonary ACC with contralateral lung metastasis.

Since primary pulmonary ACC is a low-grade malignant tumor, surgical treatment of patients with early lung adenoid cystic carcinoma is preferred. Due to the small size and complete removal of the tumors, chemotherapy and radiotherapy after complete resection is not considered. However, due to our suspicion of intrapulmonary metastatic ACC in this patient, a closer follow-up schedule based on translationally assisted by multi-omic information was prescribed. Fortunately, the patient was followed up for three years without recurrence, and the mutations detected in tumors were not found in blood by the same 425 cancer-relevant genes NGS panel.

\section{Discussion}

Primary pulmonary ACC is a low-grade malignant tumor, which usually occurs in the main bronchus, and peripheral type is rare. This patient had multiple adenoid cystic carcinomas in both lungs. But imaging and pathological examination cannot determine the relationship among the three lesions. In contrast to the diagnose of multiple pulmonary lesions with different histological types, it is still a challenge to differentially diagnose multiple lung cancers with similar histological findings. Studies have pointed out that molecular genetic analysis of gene mutation sites can assist the origin assessment. ${ }^{10}$ The shared somatic alterations in left lower and right middle lobe lesions suggesting metastases had happened, possibly driving by PIK3R1 mutation that is frequently mutated in tumors. ${ }^{11}$ Moreover, in next step we detected low PIK3R1 mRNA level in left lower lobe lesion, low PIK3R1 mRNA level is associated with high-risk for stage I non-small cell lung cancers recurrence. ${ }^{12}$ Of note, PIK3R1 mutations have 
Table I Genetic Alterations in the Three Adenoid Cystic Carcinomas

\begin{tabular}{|c|c|c|c|c|c|c|c|}
\hline Sample & $\begin{array}{c}\text { Mutation } \\
\text { Type }\end{array}$ & Gene & AAChange & Ref & Alt & $\begin{array}{c}\text { InterVar } \\
\text { Classification }\end{array}$ & Cosmic ID \\
\hline LLL & $\begin{array}{l}\text { Mutant } \\
\text { Mutant } \\
\text { Mutant } \\
\text { Mutant } \\
\text { Mutant } \\
\\
\text { CNV } \\
\text { MS } \\
\text { TMB }\end{array}$ & $\begin{array}{l}\text { PIK3RI } \\
\text { LYN } \\
\text { SPOP } \\
\text { SETD2 } \\
\text { FBXW7 } \\
\text { MCLI }\end{array}$ & $\begin{array}{c}\text { c.20Idup (p.I68Yfs*8) } \\
\text { c. 1079G >A (p.R360Q) } \\
\text { c.36IC>T (p.RI2IW) } \\
\text { c.4929C>G (p. } \\
\text { NI643K) } \\
\text { c. } 1034 \mathrm{C}>\mathrm{T} \text { (p.T345I) } \\
\text { MSS }\end{array}$ & $\begin{array}{l}- \\
G \\
G \\
G\end{array}$ & $\begin{array}{l}\mathrm{T} \\
\mathrm{A} \\
\mathrm{A} \\
\mathrm{C}\end{array}$ & $\begin{array}{l}\text { Likely pathogenic } \\
\text { Uncertain significance } \\
\text { Uncertain significance } \\
\text { Uncertain significance } \\
\text { Uncertain significance }\end{array}$ & $\begin{array}{c}\text { COSM269840I } \\
\text { COSM69934I7; COSM69934I5; } \\
\text { COSM69934I4; COSM69934I6; } \\
\text { COSM69934I 2; COSM69934I3 }\end{array}$ \\
\hline RML & $\begin{array}{c}\text { Mutant } \\
\text { MS } \\
\text { TMB }\end{array}$ & PIK3RI & $\begin{array}{c}\text { c.20Idup (p.168Yfs*8) } \\
\text { MSS }\end{array}$ & - & $\mathrm{T}$ & Likely pathogenic & \\
\hline RLL & $\begin{array}{l}\text { MS } \\
\text { TMB }\end{array}$ & & MSS & & & & \\
\hline Blood & $\begin{array}{l}\text { SNP } \\
\text { SNP } \\
\text { SNP } \\
\text { SNP } \\
\text { SNP } \\
\\
\text { SNP } \\
\text { SNP } \\
\text { TMB }\end{array}$ & $\begin{array}{c}\text { DPYD } \\
\text { ERCCI } \\
\text { ERCC2 } \\
\text { NQOI } \\
\text { TYMS } \\
\text { 6bP } \\
\text { UGTIAI } \\
\text { GSTTI }\end{array}$ & 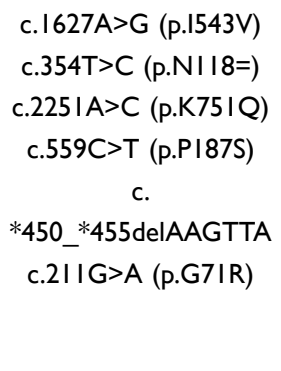 & $\begin{array}{c}\text { T } \\
\text { A } \\
\text { T } \\
\text { G } \\
\text { TTAAAG } \\
\text { G }\end{array}$ & $\begin{array}{l}C \\
G \\
G \\
A \\
-\end{array}$ & $\begin{array}{c}\text { Benign } \\
\text { Benign } \\
\text { Benign } \\
\text { Uncertain significance } \\
\text { Benign } \\
\text { Uncertain significance }\end{array}$ & $\begin{array}{l}\text { Cosm } 3747 I 21 \\
\text { cosm } 4132125 \\
\text { cosmI } 48097\end{array}$ \\
\hline
\end{tabular}

Notes: *COSMIC, the Catalogue of Somatic Mutations in Cancer; InterVar, a bioinformatics software tool for clinical interpretation of genetic variants by the ACMG/AMP 2015 guideline, defining variants into "Benign", "Likely benign", "Uncertain significance", "Likely pathogenic" and "Pathogenic", together with detailed evidence code.

been reported in a variety of tumors, understanding its prevalence and biological function is of great significance for the development of new targeted drugs in the future, such as breast cancer. ${ }^{13}$ Based on these findings, poordocumented gene expression of ACC profile, and the request of personalised post-operative decision-making, we performed second-stage investigation of this case.
In 1995, Schena et al published a report on gene chip for the first time, proving that cDNA microarray can provide a useful link between human gene expression and clinical medicine. ${ }^{14}$ Now it has been widely used in tumor research, especially in the studies of tumor metastasis. In recent years, more and more researches have applied gene expression profiling to screen pathogenic genes and to study the
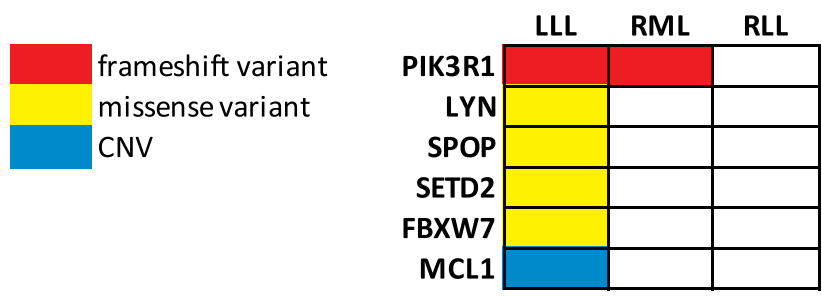

Mutation rate among 425 genes
Mutation rate among samples

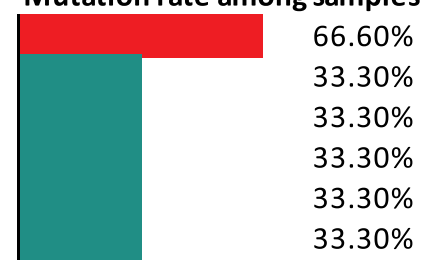

Figure I Genetic alterations of the presented multiple ACC lesions.

Abbreviations: LLL, left lower lobe; RML, right middle lobe; RLL, right lower lobe. 
A

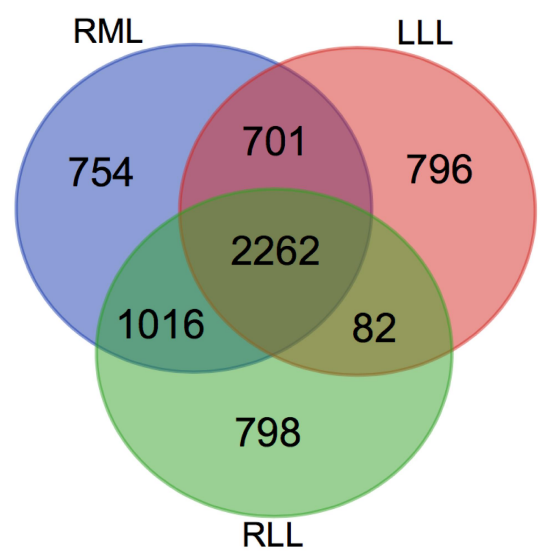

C

Cluster Dendrogram
B

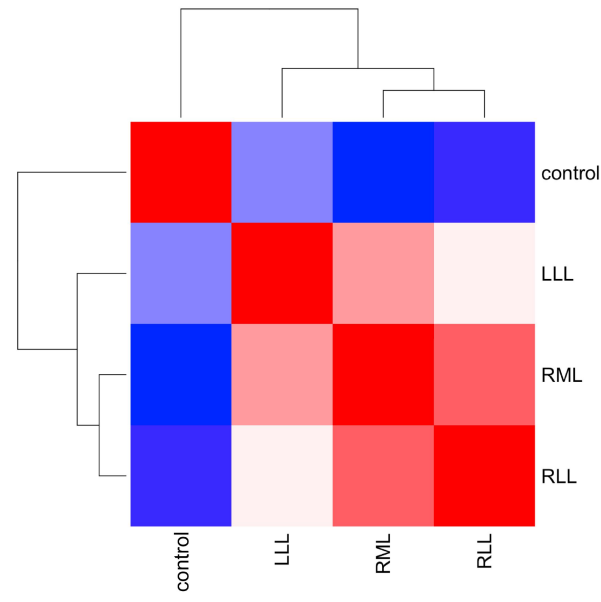

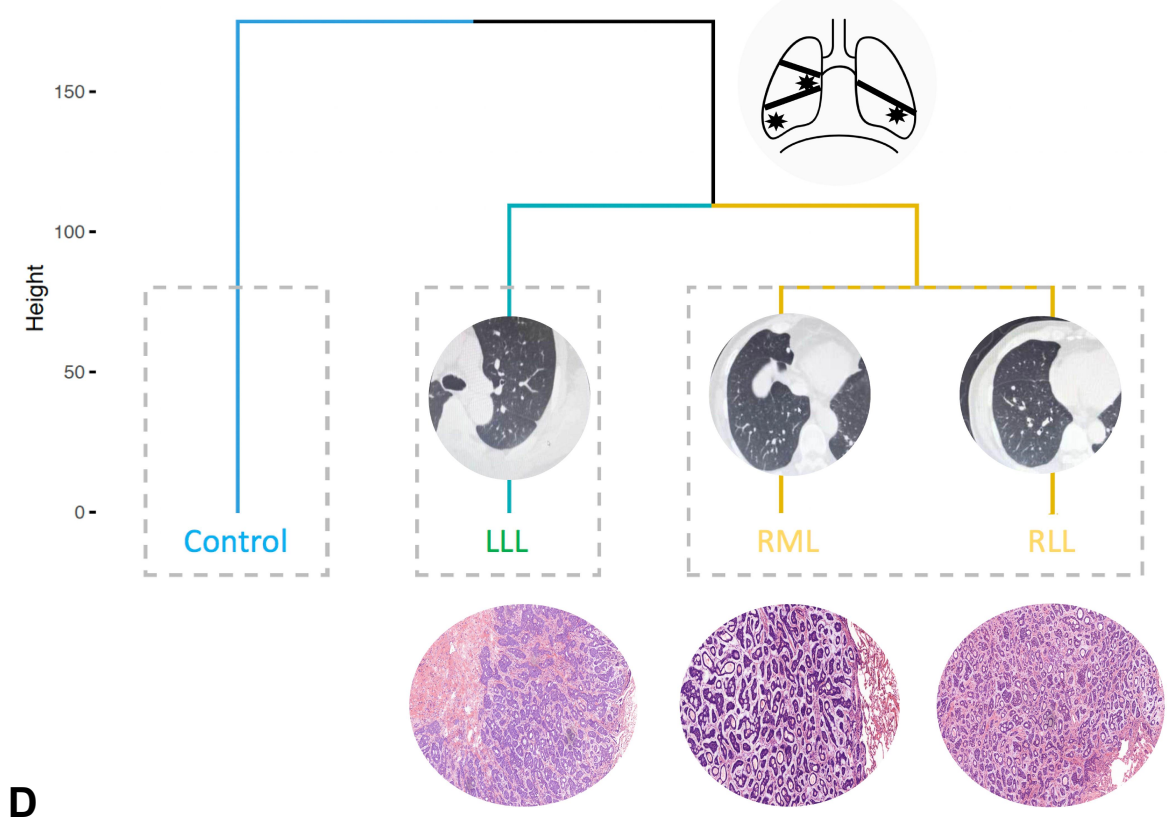

KEGG significant pathways

Protein digestion and absorption

Focal adhesion

Dilated cardiomyopathy (DCM)

Protein digestion and absorption

Human papillomavirus infection

PI3K-Akt signaling pathway

ECM-receptor interaction

Arrhythmogenic right ventricular cardiomyopathy (ARVC)

Cell adhesion molecules (CAMs)
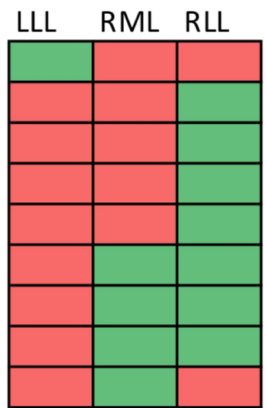

Figure 2 Transcriptome-assisted origin assessment of multiple ACC lesions. (A) The Venn diagram visualized the number of differential gene expression in the three tumors. (B) Heatmap of differential gene expression correlation matrices. (C) Hierarchical clustering and principal component analysis of gene expression profile. (D) KEGG signaling pathway analysis of DEGs in three tumors. Cells in green indicate the signaling pathways are significantly enriched, while cells in red mean the pathways are not activated.

Abbreviations: LLL, left lower lobe; RML, right middle lobe; RLL, right lower lobe; KEGG, Kyoto Encyclopedia of Genes and Genomes. 
Table 2 Overlapped Top 10 Upregulated GO Terms in the Three Malignant Tumours

\begin{tabular}{|c|c|c|c|c|c|}
\hline Term & Description & $\begin{array}{l}\text { P-value in } \\
\text { RML }\end{array}$ & $\begin{array}{c}\text { P-value in } \\
\text { RLL }\end{array}$ & $\begin{array}{c}\text { P-value in } \\
\text { LLL }\end{array}$ & $\begin{array}{c}\text { Overlap } \\
\text { Enrichment }\end{array}$ \\
\hline \multicolumn{6}{|c|}{ Biological Process } \\
\hline GO:00635I & Transcription, DNA-templated & 4.09। I 4E-06 & $3.34546 \mathrm{E}-07$ & - & Unique in right lesions \\
\hline GO:0006355 & Regulation of transcription, DNA-templated & $7.11726 \mathrm{E}-06$ & $3.61249 \mathrm{E}-07$ & - & Unique in right lesions \\
\hline GO:0002934 & Desmosome organization & $2.586 \mid 2 \mathrm{E}-05$ & 5.49273E-07 & - & Unique in right lesions \\
\hline GO:0030198 & Extracellular matrix organization & - & $9.67886 \mathrm{E}-06$ & 4.76496E-06 & Transitional \\
\hline GO:0007I55 & Cell adhesion & I.77278E-05 & $9.73916 \mathrm{E}-06$ & - & Unique in right lesions \\
\hline \multicolumn{6}{|c|}{ Molecular Function } \\
\hline GO:0003677 & DNA binding & I.78I52E-06 & $5.66698 \mathrm{E}-08$ & 0.000783362 & Common Enrichment \\
\hline GO:0000I66 & Nucleotide binding & 0.00058113 & $5.7697 \mathrm{E}-06$ & 0.002607564 & Common Enrichment \\
\hline GO:00055I5 & Protein binding & I. $16842 \mathrm{E}-09$ & $3.0942 \mathrm{E}-10$ & 7.030IIE-05 & Common Enrichment \\
\hline GO:0003700 & $\begin{array}{l}\text { Transcription factor activity, sequence-specific DNA } \\
\text { binding }\end{array}$ & $6.32364 \mathrm{E}-06$ & I.68874E-05 & 4.56837E-06 & Common Enrichment \\
\hline GO:0030020 & $\begin{array}{l}\text { Extracellular matrix structural constituent conferring } \\
\text { tensile strength }\end{array}$ & - & 0.000134592 & 0.000487548 & Transitional \\
\hline GO:0046872 & Metal ion binding & 0.001271416 & - & 0.001801895 & Transitional \\
\hline \multicolumn{6}{|c|}{ Cellular Component } \\
\hline GO:0005654 & Nucleoplasm & $8.01633 \mathrm{E}-10$ & $8.15503 \mathrm{E}-10$ & 0.002022821 & Common Enrichment \\
\hline GO:0005634 & Nucleus & $7.03725 \mathrm{E}-10$ & I.98908E-09 & $5.51924 \mathrm{E}-09$ & Common Enrichment \\
\hline GO:0045I78 & Basal part of cell & - & 0.000717997 & 0.000607947 & Transitional \\
\hline GO:007I944 & Cell periphery & - & 0.000126015 & 7.75437E-05 & Transitional \\
\hline GO:0005592 & Collagen type $X I$ trimer & $0.002324 I I I$ & - & 0.00088074 & Transitional \\
\hline GO:0005730 & Nucleolus & $1.783 \mid 3 E-07$ & 0.002198699 & - & Unique in right lesions \\
\hline
\end{tabular}

Abbreviations: GO, Gene Ontology; LLL, left lower lobe; RML, right middle lobe; RLL, right lower lobe.

mechanism of tumor metastasis. It has been reported that gene expression profiling can distinguish between primary papillary thyroid carcinoma and metastatic carcinoma, primary lung carcinomas and intrapulmonary metastases and primary and metastatic melanoma. ${ }^{7-9}$ In order to solve our real-world problems, we analyzed the gene expression profiles of three lesions in the same batch to find out the differences in gene expression levels and signal pathways among the three tumor lesions. We found that there is a significant difference in gene expression between left lower lung lesions and right-side lung lesions. At the same time, KEGG pathway analysis showed that the differential genes in the right tumor were mainly enriched in PI3K-Akt signaling pathway. It is worth mentioning that PI3K-Akt signaling pathway is closely related to tumor metastasis. Studies have shown that primary tumor cells activate this signaling pathway to cause tumor metastasis. ${ }^{15,16}$ As indicated above, we speculate that the left tumor is the primary tumor and the right lung tumors are the metastatic lesions, indicating that this patient is a primary peripheral pulmonary ACC with contralateral lung metastasis.

Adenoid cystic carcinoma of the lung is usually a long course disease, from the appearance of a lung mass to the patient's onset of symptoms, which can last for years to decades. Surgical treatment of patients with early lung adenoid cystic carcinoma has a good survival outcome. For patients who cannot receive surgery, local radiotherapy can be taken. ${ }^{17}$ In this patient, the size of the tumors were small, and we completely removed the lesions in sequential. Since the patient's lesions were completely removed, supporting by no benefits from chemotherapy and radiotherapy after complete resection of lung $\mathrm{ACC}$, the patient did not receive further treatment. Though there is still possible that metastasis from the RML lesion to the left side or synchronous lesions in both lobes, metastatic ACC from left lesion to the right side in this case was highly suspected. Therefore, a closer follow-up with liquid biopsy was suggested, ensuring free recurrence and metastasis in the next three years. 
In conclusion, studies of genetic alteration and gene expression profile are helpful to determine the relationship between multiple lung adenoid cystic carcinoma nodules, serving as a guide for precise staging and post-operative decision of synchronous bilateral of intrapulmonary metastasis of a rare malignant tumor.

\section{Abbreviations}

ACC, adenoid cystic carcinoma; GO, Gene Ontology; KEGG, The Kyoto Encyclopedia of Genes and Genomes; LLL, left lower nodule; PET/CT, positron emission tomography-computed tomography; RLL, right lower lobe; RML, right middle lobe; VATS, videoassisted thoracoscopic surgery; WHO, the World Health Organization.

\section{Data Sharing Statement}

If requested, the gene expression datasets generated for this study will be shared with patient's consensus.

\section{Ethics Statement}

This study was approved by institutional Ethics Committee of Taizhou Hospital of Zhejiang Province affiliated to Wenzhou Medical University and the patient provided the written informed consent for the publication of the case report.

\section{Author Contributions}

All authors made a significant contribution to the work reported, whether that is in the conception, study design, execution, acquisition of data, analysis and interpretation, or in all these areas; took part in drafting, revising or critically reviewing the article; gave final approval of the version to be published; have agreed on the journal to which the article has been submitted; and agree to be accountable for all aspects of the work.

\section{Funding}

This work was supported by Zhejiang Provincial Natural Foundation (LQ18H160029) and the National Natural Science Foundation of China (No. 82002420, 81902319).

\section{Disclosure}

The authors declared no conflicts of interest.

\section{References}

1. Chaudhry AP, Leifer C, Cutler LS, Satchidanand S, Labay GR, Yamane GM. Histogenesis of adenoid cystic carcinoma of the salivary glands. Light and electron microscopic study. Cancer. 1986;58 (1):72-82. doi:10.1002/1097-0142(19860701)58:1<72::AIDCNCR2820580114>3.0.CO;2-Q

2. Lawrence JB, Mazur MT. Adenoid cystic carcinoma: a comparative pathologic study of tumors in salivary gland, breast, lung, and cervix. Hum Pathol. 1982;13(10):916-924. doi:10.1016/S0046-8177(82) 80052-X

3. Travis WD, Brambilla E, Nicholson AG, et al. The 2015 World Health Organization classification of lung tumors: impact of genetic, clinical and radiologic advances since the 2004 classification. $J$ Thorac Oncol. 2015;10(9):1243-1260. doi:10.1097/JTO.0000000000000630

4. Travis WD, Travis LB, Devesa SS. Lung cancer. Cancer. 1995;75(1 Suppl):191-202.

5. Kawashima O, Hirai T, Kamiyoshihara M, Ishikawa S, Morishita Y. Primary adenoid cystic carcinoma in the lung: report of two cases and therapeutic considerations. Lung Cancer. 1998;19(3):211-217. doi:10.1016/S0169-5002(97)00098-6

6. Inoue $\mathrm{H}$, Iwashita $\mathrm{A}$, Kanegae $\mathrm{H}$, Higuchi $\mathrm{K}$, Fujinaga $\mathrm{Y}$, Matsumoto I. Peripheral pulmonary adenoid cystic carcinoma with substantial submucosal extension to the proximal bronchus. Thorax. 1991;46(2):147-148. doi:10.1136/thx.46.2.147

7. Szpak-Ulczok S, Pfeifer A, Rusinek D, et al. Differences in gene expression profile of primary tumors in metastatic and non-metastatic papillary thyroid carcinoma-do they exist? Int J Mol Sci. 2020;21 (13):4629. doi:10.3390/ijms21134629

8. Chang JC, Alex D, Bott M, et al. Comprehensive next-generation sequencing unambiguously distinguishes separate primary lung carcinomas from intrapulmonary metastases: comparison with standard histopathologic approach. Clin Cancer Res. 2019;25(23):7113-7125. doi:10.1158/1078-0432.CCR-19-1700

9. Li Y, Krahn JM, Flake GP, Umbach DM, Li L. Toward predicting metastatic progression of melanoma based on gene expression data. Pigment Cell Melanoma Res. 2015;28(4):453-463. doi:10.1111/ pcmr. 12374

10. Tan B, Jiang X, Wang R, et al. Genomic profiling reveals synchronous bilateral lung adenocarcinomas with distinct driver alterations of EML4-ALK or TPM3-ROS1 fusion: a case report. Front Oncol. 2019;9:1319. doi:10.3389/fonc.2019.01319

11. Costa C, Engelman JA. The double life of p85. Cancer Cell. 2014;26 (4):445-447. doi:10.1016/j.ccell.2014.09.011

12. Lu Y, Lemon W, Liu PY, et al. A gene expression signature predicts survival of patients with stage I non-small cell lung cancer. PLoS Med. 2006;3(12):e467. doi:10.1371/journal.pmed.0030467

13. Chen L, Yang L, Yao L, et al. Characterization of PIK3CA and PIK3R1 somatic mutations in Chinese breast cancer patients. Nat Commun. 2018;9(1):1357. doi:10.1038/s41467-018-03867-9

14. Schena M, Shalon D, Davis RW, Brown PO. Quantitative monitoring of gene expression patterns with a complementary DNA microarray. Science. 1995;270(5235):467-470. doi:10.1126/ science. 270.5235 .467

15. Bakin AV, Tomlinson AK, Bhowmick NA, Moses HL, Arteaga CL. Phosphatidylinositol 3-kinase function is required for transforming growth factor beta-mediated epithelial to mesenchymal transition and cell migration. $J$ Biol Chem. 2000;275(47):36803-36810. doi:10.1074/jbc.M005912200

16. Porta C, Paglino C, Mosca A. Targeting PI3K/Akt/mTOR signaling in cancer. Front Oncol. 2014;4:64. doi:10.3389/fonc.2014.00064

17. Bonner Millar LP, Stripp D, Cooper JD, Both S, James P, Rengan R. Definitive radiotherapy for unresected adenoid cystic carcinoma of the trachea. Chest. 2012;141(5):1323-1326. doi:10.1378/chest.110925 


\section{Publish your work in this journal}

Cancer Management and Research is an international, peer-reviewed open access journal focusing on cancer research and the optimal use of preventative and integrated treatment interventions to achieve improved outcomes, enhanced survival and quality of life for the cancer patient.

Submit your manuscript here: https://www.dovepress.com/cancer-management-and-research-journa
The manuscript management system is completely online and includes a very quick and fair peer-review system, which is all easy to use. Visit http://www.dovepress.com/testimonials.php to read real quotes from published authors. 